\title{
Serum level of oxidative stress marker is dramatically low in patients with rheumatoid arthritis treated with tocilizumab
}

\author{
Makoto Hirao • Naomi Yamasaki • Hiroki Oze $\cdot$ Kosuke Ebina • \\ Akihide Nampei $\cdot$ Yoshitaka Kawato $\cdot$ Kenrin Shi $\cdot$ Hideki Yoshikawa $\cdot$ \\ Norihiro Nishimoto $\cdot$ Jun Hashimoto
}

Received: 4 May 2011/Accepted: 21 August 2011/Published online: 11 September 2011

(C) The Author(s) 2011. This article is published with open access at Springerlink.com

\begin{abstract}
Regarding the pathobiology of rheumatoid arthritis, oxidative stress induced by reactive oxygen species is an important mechanism that underlies destructive and proliferative synovitis. Abundant amounts of reactive oxygen species have been detected in the synovial fluid of inflamed rheumatoid joints. It is reported that drugs that block tumor necrosis factor- $\alpha$ reduce the oxidative stress marker levels in patients with rheumatoid arthritis. In this study, we measured reactive oxygen species using a free radical analytical system in patients with rheumatoid arthritis treated with disease-modifying antirheumatic drugs, tumor necrosis factor- $\alpha$-blocking drugs (infliximab, etanercept), and an interleukin-6-blocking drug (tocilizumab). The serum level of oxidative stress was drastically low in patients with rheumatoid arthritis treated with tocilizumab, suggesting that interleukin-6 blocking therapy reduces not only joint damage, but also vascular degeneration in patients with rheumatoid arthritis. We believe that such a drastic effect would reduce the incidence of cardiovascular events and mortality in patients with rheumatoid arthritis.
\end{abstract}

M. Hirao · N. Yamasaki · H. Oze - K. Ebina - A. Nampei ·

Y. Kawato - K. Shi · H. Yoshikawa · J. Hashimoto

Department of Orthopaedics, Osaka University Graduate School

of Medicine, Osaka, Japan

N. Nishimoto

Laboratory of Immune Regulation, Wakayama Medical School,

Wakayama, Japan

J. Hashimoto $(\square)$

Department of Immune Disease Center, National Hospital

Organization, Osaka Minami Medical Center, 2-1 Kidohigashi,

Kawachinagano City, Osaka 586-8521, Japan

e-mail: junha89@gmail.com
Keywords Rheumatoid arthritis - Oxidative stress · Reactive oxygen species $\cdot$ IL- 6 - Tocilizumab

\section{Introduction}

Rheumatoid arthritis (RA) is a chronic inflammatory disease of unknown etiology and affects synovial joints, leading to the development of cartilage and subchondral bone erosion [1]. Regarding the pathobiology of RA, oxidative stress induced by reactive oxygen species (ROS) is an important mechanism that underlies destructive and proliferative synovitis [2-5]. Reactive oxygen species are produced during oxidative phosphorylation. When the production of ROS exceeds the physiological limit, it induces oxidative stress and damages proteins, lipids, and nucleic acids. Abundant amounts of ROS have been detected in the synovial fluid of inflamed rheumatoid joints [6], and this abundance can act as a second messenger to stimulate nuclear factor kappa B-dependent expression of pro-inflammatory cytokines and form an amplification loop that feeds back to further excite the production of additional ROS. The production of ROS can be induced by TNF- $\alpha$ stimulation [7, 8]. In inflammatory arthritis, including RA, TNF- $\alpha$ has been shown to produce ROS from neutrophils. Therefore, TNF- $\alpha$-blocking drugs should have an effect on ROS generation. In fact, it is reported that infliximab reduces the oxygen stress markers, including pentosidine and 8-hydroxy-2'-deoxyguanosine [9]. It is also reported that protein carbonyl groups are decreased and thiols are increased by infliximab treatment, indicating that serum protein oxidation is suppressed. Etanercept also blocks TNF- $\alpha$, although the action of this drug is slightly different from that of infliximab. Etanercept also reduces the oxidative stress marker levels in patients with RA [10]. 
From these observations, TNF- $\alpha$-blocking therapy appears to reduce oxidative stress in patients with RA.

On the other hand, IL-6 is also a pivotal pro-inflammatory cytokine in RA and IL-6 reportedly induces ROS production in RA synovial fibroblastic cells [11]. Thus, IL-6 blocking treatment may also suppress the oxidative stress in patients with RA. However, no study to date has reported this. In this study, we evaluated the effect of tocilizumab, which is a novel IL-6-blocking biologics, on the serum ROS levels in patients with RA. Direct measurement of ROS and free radicals in standard laboratories is difficult because of their biochemical instability. Recently, a method of measuring reactive oxygen metabolites (ROM) in blood has been developed. This method is the d-ROM test, which uses the Free Radical Analytical System (FRAS, Wismarl, Italy) [12, 13]. The main component of ROM is hydroperoxides. Despite their fair oxidant power, hydroperoxides in the blood are relatively stable compared to their parent-free radicals, so their level can be detected. Hydroperoxides cause cell death and tissue damage. The d-ROM test has already been recognized as useful for the evaluation of oxidative stress in the body $[14,15]$. In this study, we measured ROS using a free radical analytical system (FRAS4) in patients with RA treated with disease-modifying antirheumatic drugs (DMARDs), TNF- $\alpha$-blocking drugs (infliximab, etanercept), and an IL-6-blocking drug (tocilizumab).

\section{Methods}

Patients and blood samples

We investigated 140 patients including 15 patients with osteoarthritis (OA), 91 patients with RA treated with
DMARDs, 8 patients with RA treated with tumor necrosis factor-blocking biologics (infliximab, etanercept), and 26 patients with RA treated with an IL-6-blocking biologic (tocilizumab). All of the patients who received biologics treatment received it for at least 3 months. This observational study started after approval by the Institutional Review Board at the Osaka University Hospital. Sampling was performed from all patients who consent to the blood sampling for study among all patients with RA or OA visited our clinic during 2 months that was from September to October 2008. Venous blood samples were collected in tubes and analyzed for serum C-reactive protein (CRP) and white blood cell count. In our hospital, the normal reference value for CRP is $<0.2 \mathrm{mg} / \mathrm{dl}$. Reactive oxygen species was also measured as described below (Table 1).

\section{Measurement of oxidative stress marker in serum}

As previously mentioned, direct measurement of ROS and free radicals in standard laboratories is difficult because of their biochemical instability. Recently, a method of measuring ROM in blood has been developed. To measure ROM, the d-ROM test was performed using the FRAS4 analyzer (Wismarl, Italy), according to the analysis procedures. In brief, a $20 \mu \mathrm{l}$ blood sample and $1 \mathrm{~mL}$ of buffered solution ( $\mathrm{R} 2$ reagent of kit, $\mathrm{pH}$ 4.8) were gently mixed in a cuvette, and then $10 \mu \mathrm{l}$ of chromogenic substrate (R1 reagent of kit) was added into the cuvette. After mixing, the cuvette was centrifuged for $60 \mathrm{~s}$ at $37^{\circ} \mathrm{C}$ and immediately incubated in the thermostatic block of this analyzer for $5 \mathrm{~min}$ at $37^{\circ} \mathrm{C}$. Then, the absorbance at $505 \mathrm{~nm}$ was recorded. The measurement unit was expressed as Carr U. It has been established that 1 Carr U corresponds to $0.08 \mathrm{mg} / \mathrm{dl}_{2} \mathrm{O}_{2}$. Reference values indicated

Table 1 Characteristics of patient backgrounds regarding age, duration of disease, body weight, volume of daily intake of prednisolone, C-reactive protein (CRP) levels, white blood cell counts, and reactive oxygen metabolite levels

\begin{tabular}{llccc}
\hline & Control $(\mathrm{OA}) N=15$ & \multicolumn{2}{l}{ RA } & \\
\cline { 3 - 5 } & & DMARDs $N=91$ & Anti-TNF $N=8$ & Anti-IL-6 $N=26$ \\
\hline Age (years) & $68.1 \pm 14.5$ & $63.1 \pm 12.1$ & $58.8 \pm 3.81$ & $58.0 \pm 8.0$ \\
Duration of disease (years) & - & $12.6 \pm 10.7$ & $10.3 \pm 4.5$ & $13.0 \pm 8.5$ \\
Body weight (kg) & - & $51.4 \pm 9.6$ & $54.9 \pm 7.9$ & $52.8 \pm 7.7$ \\
Prednisolone (mg/day) & 0 & $2.9 \pm 3.1$ & $2.8 \pm 3.2$ & $2.0 \pm 3.0$ \\
CRP (mg/d) & - & $1.12 \pm 1.76$ & $0.42 \pm 0.37^{*}$ & $0.03 \pm 0.14^{* *}$ \\
WBC (counts) & - & $8,090 \pm 2,914$ & $7,746 \pm 2,779$ & $6,302 \pm 2,866$ \\
ROM (d-ROM: Carr U) & $375.5 \pm 72.5^{*}$ & $464.2 \pm 112.3$ & $377.1 \pm 53.7^{*}$ & $239.2 \pm 73.7^{* *}$ \\
\hline
\end{tabular}

There was no significant difference in disease duration, body weight, daily dose of prednisolone, and WBC counts in among the three groups of RA. CRP level was significantly lower in anti-IL-6 (TCZ) group than other two RA groups and that of anti-TNF group was significantly lower than DMARDs group but significantly higher than anti-IL-6 group

*, ** $P<0.05$, significantly lower level of CRP level and d-ROM as compared with that in the DMARD group. Results are expressed as the mean $\pm \mathrm{SD}$. Between-group differences were assessed by one-way ANOVA test, and a probability $<0.05$ was considered to indicate statistical significance 
by the manufacturer (Wismarl) are from <300 Carr U; values higher than 300 Carr U suggest oxidative stress [12, 13].

\section{Statistical analysis}

Results are expressed as the mean \pm SD. Between-group differences were assessed by one-way ANOVA test, and a probability $<0.05$ was considered to indicate statistical significance. All statistical analysis was performed using SPSS (ver. 11.5) software (SPSS, IL, USA).

\section{Results}

\section{Background characteristics}

OA group was older than other three groups of RA, and DMARDs group was older than other two biologics groups, however, there was no significant difference in age among 4 groups. There was no significant difference in disease duration, body weight, daily dose of prednisolone and WBC counts in among the three groups of RA. CRP level was significantly lower in anti-IL-6 (TCZ) group $(0.03 \pm 0.14)$ than other two RA groups and that of antiTNF group $(0.42 \pm 0.37)$ was significantly lower than DMARDs group $(1.12 \pm 1.76)$ but significantly higher than anti-IL-6 group (Table 1).

Serum levels of oxidative stress is significantly increased in patients with RA

As shown in Fig. 1, 15 patients with OA showed $375.5 \pm 72.5$ Carr U of ROM, while 91 patients with RA treated with DMARDs showed 464.2 \pm 112.3 Carr U of ROM; all but four of the patients in the latter group showed abnormal levels (>300 Carr U). Levels of ROM were significantly higher in the RA group treated with DMARDS rather than biologics, suggesting that oxidative stress is up-regulated in the pathobiology of RA. The value of $375.5 \pm 72.5$ Carr $U$ in the OA group is also increased from the normal limit (<300 Carr U).

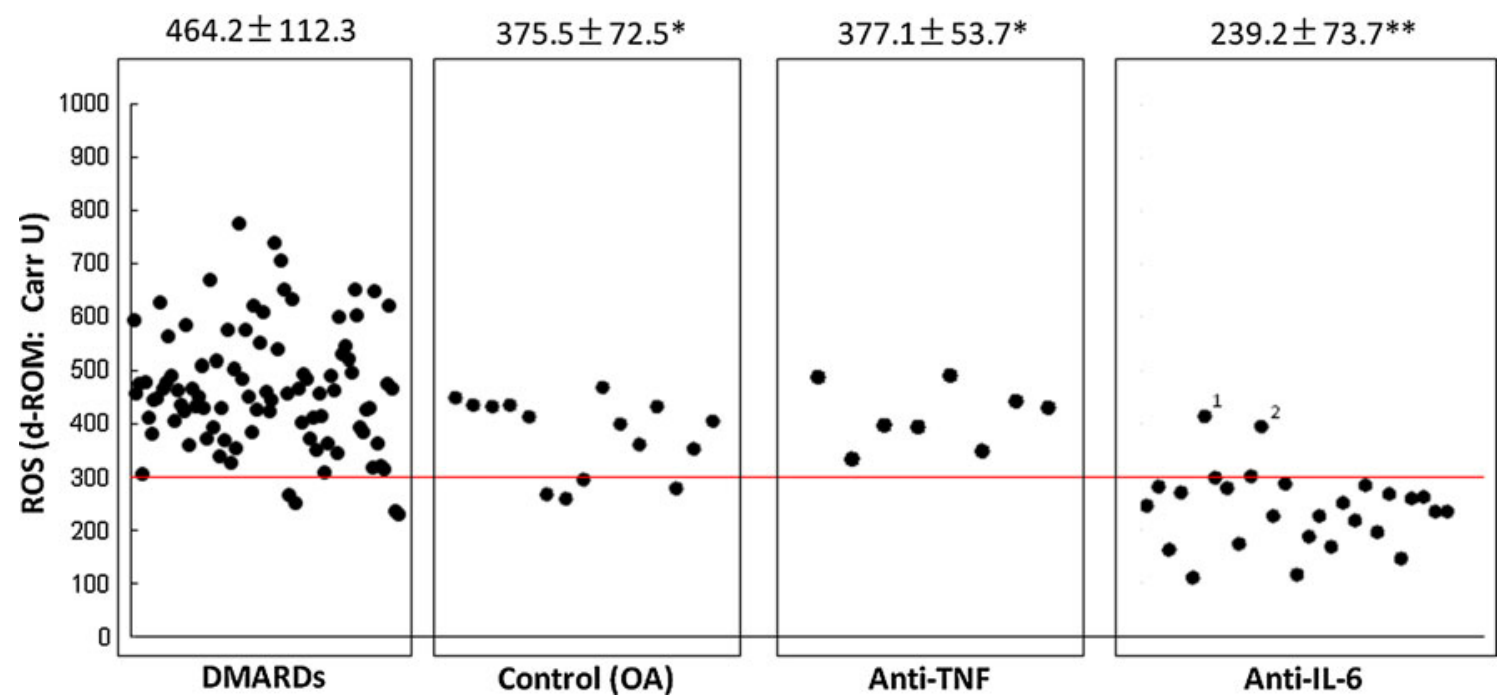

Fig. 1 Serum levels of oxidative stress are significantly increased in patients with RA. Fifteen patients with OA showed $375.5 \pm 72.5$ Carr $\mathrm{U}$ of ROM, while 91 patients with rheumatoid arthritis treated with disease-modifying antirheumatic drugs showed 464.2 \pm 112.3 Carr U of ROM, and all but four of these patients showed abnormal levels ( $>300$ Carr U). Levels of ROM were significantly higher in the group of patients with rheumatoid arthritis not treated with biologics, suggesting that oxidative stress is up-regulated in the pathobiology of rheumatoid arthritis. The value of $375.5 \pm 72.5$ Carr $U$ in the OA group is increased as compared with the normal limit ( $<300$ Carr U). However, this seems to be due to aging. Serum level of oxidative stress markers is significantly low in patients with RA treated with anti-TNF biologics therapy. Serum levels of ROM in 8 patients with rheumatoid arthritis treated with tumor necrosis factor-blocking therapy showed $377.1 \pm 53.7$ Carr $\mathrm{U}$ of ROM, and these levels are similar to those in the OA group, suggesting that anti-TNF biologics therapy effectively reduces oxidative stress in patients with rheumatoid arthritis. Serum level of oxidative stress is dramatically low in patients with rheumatoid arthritis treated with anti-IL-6 biologics therapy. Serum levels of ROM in 26 patients with rheumatoid arthritis treated with IL-6-blocking therapy showed $239.2 \pm 73.7$ Carr U of ROM. Only two cases (Cases 1 and 2) showed abnormal levels (408 and 394 Carr U). Furthermore, the C-reactive protein levels of these two cases were not completely suppressed $(0.7$ and $0.1 \mathrm{mg} / \mathrm{dl})$. On the other hand, the C-reactive protein levels of the 24 cases showing normal levels of ROM were completely suppressed $(<0.04 \mathrm{mg} / \mathrm{dl})$. We could confirm that the serum levels of oxidative stress markers are dramatically low in patients with rheumatoid arthritis treated with anti-IL-6 biologics therapy, suggesting that tocilizumab has a very strong effect of reducing oxidative stress in patients with rheumatoid arthritis. ${ }^{*}, * * P<0.05$, significantly lower level d-ROM as compared with that in the DMARD group. Results are expressed as the mean \pm SD. Between-group differences were assessed by one-way ANOVA test, and a probability $<0.05$ was considered to indicate statistical significance 
Serum level of oxidative stress is significantly low in patients with RA treated with anti-TNF biologics therapy

As shown in Fig. 1, serum levels of ROM in 8 patients with RA treated with TNF-blocking therapy showed $377.1 \pm 53.7$ Carr U of ROM, a level similar to that in the OA group ( $375.1 \pm 72.5)$, suggesting that anti-TNF biologics therapy effectively reduces oxidative stress in patients with RA.

Serum level of oxidative stress is dramatically low in patients with RA treated with anti-IL-6 biologics therapy

As shown in Fig. 1, serum levels of ROM in 26 patients with RA treated with IL-6 blocking therapy showed $239.2 \pm 73.7$ Carr U of ROM, and only two cases were outside normal limits (409 and 394 Carr U). Furthermore, the CRP levels of these two cases were not completely suppressed $(0.7$ and $0.1 \mathrm{mg} / \mathrm{dl})$; on the other hand, the CRP levels of the 24 cases showing normal levels of ROM were completely suppressed $(<0.04 \mathrm{mg} / \mathrm{dl})$. We could confirm that the serum levels of oxidative stress are significantly and dramatically low in patients with RA treated with antiIL-6 biologics therapy, suggesting that tocilizumab has a very strong effect of reducing oxidative stress in patients with RA.

\section{Discussion}

This paper is the first report about the influence of IL-6 blocking therapy using tocilizumab on oxidative stress in patients with RA. The serum level of oxidative stress markers was significantly low in patients treated with tocilizumab. We observed not only this drastic effect of IL6-blocking biologics for the suppression of oxidative stress, but also the up-regulation of oxidative stress in the pathophysiology of RA and its significant suppression by TNF antagonists as described previously.

Oxidative stress induced by ROS is an important mechanism that underlies destructive and proliferative synovitis [2-5]; it is also a very important mediator of extra-articular diseases of RA. Rheumatoid arthritis is associated with increased cardiovascular morbidity and mortality [16]. Patients with RA have a higher risk of cardiovascular events not explained entirely by traditional cardiovascular risk factors [17], implying that cardiovascular disease is an extra-articular manifestation of RA [18]. In the pathobiology of cardiovascular events, arterial atherosclerosis exists and increased arterial stiffness, increased carotid intima media thickness, and endothelial dysfunction have been demonstrated in patients at increased risk of and with known cardiovascular disease [19, 20], and these pathological changes in cardiovascular system have been also reported to be observed in patients with RA $[21,22]$. In the process of arterial atherosclerosis, oxidative stress is a very important trigger as described previously. Then, it is plausible that the suppression of oxidative stress would decrease the risk for atherosclerosis and calcification of the arteries followed by a decrease in cardiovascular events, resulting in an improvement in mortality in patients with RA.

Indeed, TNF-blocking biologics are confirmed not only to reduce RA-induced oxidative stress in several previous reports and in this study, but also to improve vascular stiffness in patients with RA [23]. In this study, we could confirm the dramatically lower oxidative stress in IL-6blocking biologics users, so tocilizumab is expected to also improve not only intra-articular synovitis and destruction, but also arterial atherosclerosis, an extra-articular manifestation. Subsequently, we must check the effect of tocilizumab on vascular stiffness and intima media thickness. In this study, 2 cases with positive CRP level under the TCZ administration showed the oxidative stress higher than upper limit of normal range although all other cases with negative CRP level have oxidative stress lower than normal upper limit. This findings also suggest that block of IL-6 signal induce the suppression of oxidative stress. Samples in this study are obtained from all cases in a fixed period, and we did not match the age or duration of RA before sampling. However, there was no significant difference in age and duration of RA among three groups in RA. Age of OA groups was older than RA groups although there was no significance possibly due to small number, so there might be age-related bias in ROM in OA group because oxidative stress is influenced by age [24, 25]. This study has several limitations due to the cross-sectional observational study. We did not show the longitudinal change in oxidative stress induced by introduction of TCZ. A prospective study would confirm the reduction in oxidative stress by TCZ with its time course and its variation among the cases. Another limitation is the lack of sufficient number of patients treated with TNF-alpha blockers to evaluate those effects on oxidative stress and the further study is required.

In conclusion, the serum level of oxidative stress markers is drastically low in patients with RA treated with tocilizumab, suggesting that IL-6 blocking therapy reduces not only joint damage, but also vascular degeneration in patients with RA. We believe that this drastic effect may reduce the incidence of cardiovascular events and mortality in patients with RA. 
Open Access This article is distributed under the terms of the Creative Commons Attribution Noncommercial License which permits any noncommercial use, distribution, and reproduction in any medium, provided the original author(s) and source are credited.

\section{References}

1. Pap T, Muller-Ladner U, Gay RE, Gay S (2009) Fibroblast biology: role of synovial fibroblasts in the pathogenesis of rheumatoid arthritis. Arthritis Res 2:361-367

2. Mapp PI, Grootveld MC, Blake DR (1995) Hypoxia, oxidative stress, and rheumatoid arthritis. Br Med Bull 51:419-436

3. Hitchon CA, El-Gabalawy H (2004) Oxidation in rheumatoid arthritis. Arthritis Res Ther 6:265-278

4. Halliwell B (1995) Oxygen radicals, nitric oxide, and human inflammatory joint disease. Ann Rheum Dis 54:505-510

5. Tak PP, Zvaifler NJ, Grenn DR, Firestein GS (2000) Rheumatoid arthritis and p53: how oxidative stress might alter the course of inflammatory diseases. Immunol Today 21:78-82

6. Biemond P, Swaak AJ, Koster JF (1984) Protective factors against oxygen free radicals and hydrogen peroxide in rheumatoid arthritis synovial fluid. Arthritis Rheum 27:760-765

7. Woo CH, Kim TH, Choi JA et al (2006) Inhibition of receptor internalization attenuates the TNF alpha-induced ROS generation in non-phagocytic cells. Biochem Biophys Res Commun 351:972-978

8. Sakon S, Xue X, Takekawa M et al (2003) NF-kappaB inhibits TNF-induced accumulation of ROS that mediate prolonged MAPK activation and necrotic cell death. EMBO J 22:3898-3909

9. Kageyama Y, Takahashi M, Ichikawa T, Torikai E, Nagano A (2008) Reduction of oxidative stress marker levels by anti-TNFalpha antibody, infliximab, in patients with rheumatoid arthritis. Clin Exp Rheumatol 26(1):73-80

10. Kageyama Y, Takahashi M, Nagafusa T, Torikai E, Nagano A (2008) Etanercept reduces the oxidative stress marker levels in patients with rheumatoid arthritis. Rheumatol Int 28:245-251

11. Sung JY, Hong JH, Kang HS, Choi I, Lim SD, Lee JK, Seok LH, Lee JH, Hur GM (2000) Methotrexate suppresses the interleukin6 induced generation of reactive oxygen species in the synoviocytes of rheumatoid arthritis. Immunopharmacology 47:35-44

12. Carratelli M, Porcaro L, Ruscica M, De Simone E, Bertelli AAE, Corsi MM (2001) Reactive oxygen metabolites and prooxidant status in children with Down's syndrome. Int J Clin Pharm Res 21(2):79-84

13. Trotti R, Carratelli M, Barbieri M, Micieli G, Bosone D, Rondanelli M, Bo P (2001) Oxidative stress and a thrombophilic condition in alcoholics without severe liver disease. Haematologica 86:85-91
14. Ippolito $\mathrm{S}$, Caruso R, Novembrino C, Bamonti-Catena F, Della Noce C, Lorenzano E, Campolo J, Tonini A, Lonati S, Rosina M, Accinni R (2002) Dislipidemia and oxidative stress. Italian J Biochem 51:129-139

15. Gerardi G, Usberti M, Martini G, Albertini A, Sugherini L, Pompella A, Lorenzo DD (2002) Plasma total antioxidant capacity in hemodialyzedpatients and its relationships to other biomarkers of oxidative stress and lipid peroxidation. Clin Chem Lab Med 40:104-110

16. Jacobsson LT, Turesson C, Hanson RL, Pillemer S, Sievers ML, Pettitt DJ et al (2001) Joint swelling as a predictor of death from cardiovascular disease in a population study of Pima Indians. Arthritis Rheum 44:1170-1176

17. Del Rincon I, Williams K, Stern MP, Freeman GL, Escalante A (2001) High incidence of cardiovascular events in a rheumatoid arthritis cohort not explained by traditional cardiac risk factors. Arthritis Rheum 44:2737-2745

18. Van Doornum S, McColl G, Wicks IP (2002) Accelerated atherosclerosis: an extraarticular feature of rheumatoid arthritis? Arthritis Rheum 46:862-873

19. Kobayashi K, Akishita M, Yu W, Hashimoto M, Ohni M, Toba K (2004) Interrelationship between non-invasive measurements of atherosclerosis: flow-mediated dilation of brachial artery, carotid intima-media thickness, and pulse wave velocity. Atherosclerosis 173:13-18

20. O'Leary DH, Polak JF, Kronmal RA, Manolio TA, Burke GL, Wolfson SKJ, Cardiovascular Health Study Collaborative Research Group (1999) Carotid-artery intima and media thickness as a risk factor for myocardial infarction and stroke in older adults. N Engl J Med 340:14-22

21. Boutouyrie P, Tropeano AI, Asmar R, Gautier I, Benetos A, Lacolley P et al (2002) Aortic stiffness is an independent predictor of primary coronary events in hypertensive patients: a longitudinal study. Hypertension 39:10-15

22. Del Porto F, Lagana B, Lai S, Nofroni I, Tinti F, Vitale M et al (2007) Response to anti-tumour necrosis factor alpha blockade is associated with reduction of carotid intima-media thickness in patients with active rheumatoid arthritis. Rheumatology 46:1111-1115

23. Angel K, Provan SA, Gulseth HL, Mowinckel P, Kvien TK, Atar D (2010) Tumor necrosis factor- $\alpha$ antagonists improve aortic stiffness in patients with inflammatory arthropathies: a controlled study. Hypertension 55:333-338

24. Agarwal S, Sohal RS (1994) Aging and protein oxidative damage. Mech Ageing Dev 75:11-19

25. Ames BN, Shigenaga MK, Hagen TM (1993) Oxidants, antioxidants, and the degenerative diseases of aging. Proc Natl Acad Sci USA 90:7915-7922 